\title{
Capacity Fade Modeling of Li-Ion Battery using Evolutionary Algorithm
}

\author{
Tamilselvi $\mathrm{S}^{1^{*}}$, Karuppiah $\mathrm{N}^{2}$, and Rajagopal Reddy $\mathrm{B}^{3}$ \\ ${ }^{1}$ Associate Professor, Department of Electrical and Electronics Engineering, SSN College of Engineering, Chennai, Tamilnadu, India, \\ tamilselvis@ssn.edu.in \\ ${ }^{2}$ Professor, Department of Electrical and Electronics Engineering, Vardhaman College of Engineering, Shamshabad, Hyderabad, India, \\ natarajankaruppiah@gmail.com \\ ${ }^{3}$ Associate Professor, Department of Electrical and Electronics Engineering, Vardhaman College of Engineering, Shamshabad, Hyderabad, \\ India, reddy.rajagopal@gmail.com
}

\begin{abstract}
Renewable sources are seasonal and cannot be considered as available energy source as their generation varies with time. The insufficient forecasting techniques lead to thought of storage of energy. Even though many techniques of energy storage are available, batteries play a vital role as the time taken to start delivering the stored energy is very less. The life period of the battery depends upon the charging and discharging characteristics which in turn depend on the internal parameters such as life period, charge rate, discharge rate of the battery. The energy stored in the battery can be calculated by finding these parameters. In this paper these parameters are estimated for a Sony lithium ion battery by evolutionary algorithm CMAES under different Charging and discharging rates. As the batteries are charged and discharged there is capacity loss in the battery. This loss is modelled by modified Arrhenius equation on practical conditions. Capacity loss of the sample battery is modelled for five different cycles starting from 50th cycle to 100th cycle in an interval of 10 cycles. The results are validated with those of manufacturer catalogue. The optimized battery capacity loss are found to coincide with the measured values.
\end{abstract}

\section{Introduction}

The demand for electrical energy has increased at a rate of $6 \%$ per annum over the past 10 years and the approximate per capita energy consumption is $1075 \mathrm{KWh}$ [1] in India. During peak hours, the demand is $159 \mathrm{GW}$ against availability of $156 \mathrm{GW}$ which is a shortage of $3.2 \%$ [2]. The rapid growth in the field of renewable energy resources has increased the overall installed capacity of the country. But these renewable energy sources are seasonal and cannot be considered as available energy source. Insufficient forecasting techniques also lead to thought of using energy storage device (ESD) at available period. From the power plant usage data it is seen that most of the power plants are underutilized during the off-peak hours. If some energy storage device can be used to store the energy during the off-peak hours, then an energy gap of $500 \mathrm{MW}$ during peak power demand can be met. Therefore, an Energy Storage System (ESS) is required to solve the issue of energy gap during peak hours. In order to handle the peak power demand, one possible solution can be to ramp-up the power generation capacity, but this option would require significant infrastructure cost of the power plants.

Even though many techniques of energy storage are available, batteries play a vital role and the time taken to deliver the stored energy is very less. But the efficiency of the available batteries in the market is very less. Even though these existing batteries are charged during off peak hours, the extracted energy transfer from batteries to grid during peak demand hours may not be sufficient. The reason for this issue is that the available power in the batteries is dependent on the characteristics of the battery. Every battery has its own characteristics and the battery characteristics depend on the internal parameters such as resistance, capacitance and the open circuit voltage. These internal parameters vary with respect to life period, charge rate, discharge rate of the battery. The frequent charging and discharging of batteries, to solve the power shortage issue, result in the degradation of the internal circuit parameters of these batteries [3, 4]. The parameters such as state of charge, depth of discharge, charge rate and discharge rate get affected. Due to these problems the capacity of the batteries gets decreased. This degradation in capacity of the battery is proportional to charge or discharge rate and temperature. Thus the life period of the battery depends upon the charging and discharging characteristics of the battery. Hence it is necessary to study and analyze the battery characteristics for increasing efficiency.

The different types of batteries used are lead acid, lithiumion, alkaline battery etc. Lithium ion batteries are the most efficient of all [5].There are four types of battery models

\footnotetext{
* Corresponding author: tamilselvi.manjuraj@gmail.com
} 
available. They are Electro-chemical models, Experimental models, Mathematical models and Electric circuit models. Of all these four, electric circuit models are best suited to represent the characteristics of EV battery. Wardburg impedance model is taken as the base model. It consists of an open circuit voltage in series with resistance and parallel RC circuit. To model a battery the internal circuit parameters are to be estimated. But the estimation of internal circuit parameters in a battery is tedious due to their non-linear behaviour. Soft computing techniques are used nowadays to extract the parameters of the battery.

Praveen Kumar, Pavol Bauser presented a paper "Parameter extraction of Battery Models Using Multi objective Optimization Genetic Algorithms"-14 ${ }^{\text {th }}$ International Power Electronics and motion Control Conference $-2010[6]$. In this work a model of the battery and a methodology for determining the parameters of a battery model using the catalogue data of the batteries. An Battery Model (BM) and Capacity Model (CM) was done by Kannan Thirugnanam, Himanshu Saini and Praveen Kumar Indian Institute of Technology Guwahati/Electronics and Electrical Engineering, Guwahati-781039, India, in their paper "Mathematical Modelling of Li-Ion Battery for Charge/Discharge Rate and Capacity Fading Characteristics using Genetic Algorithm Approach." And published in IEEE journal 2012 [7]. Using Battery modelling and Capacity Modelling, the amount of capacity fading is analyzed while the power is stored in the battery or transferred to the grid. The BM is developed for Li-ion battery based on the Electrical Equivalent Circuit (EEC) and the CM is proposed based on processed energy for different charging/discharging rate. The proposed model is simulated using MATLAB Simulink environment and the parameters are optimized using binary Genetic Algorithm (GA) approach to compare the results with manufacturer's catalogue data. In 2014, in the paper "Mathematical Modelling of Li-Ion Battery Using Genetic Algorithm Approach for V2G Applications", Kannan Thirugnanam, Student Member, IEEE, Ezhil Reena Joy T. P., Student Member, IEEE, Mukesh Singh, Student Member, IEEE, and Praveen Kumar, Member, IEEE- IEEE transactions on Energy conversions-2014 [8] presents an electric circuit-based battery and a capacity fade model suitable for electric vehicles (EVs) invehicleto-grid applications. The circuit parameters of the battery model (BM) are extracted using genetic algorithm-based optimization method. A control algorithm has been developed for the battery, which calculates the processed energy, charge or discharge rate, and state of charge limits of the battery in order to satisfy the future requirements of EVs. A complete capacity fade analysis has also been carried out to quantify the capacity loss with respect to processed energy and cycling. Min Chen, Student Member IEEE and Gabriel A. Rincon-Mora, Senior Member, IEEE published a paper "Accurate Electrical Battery Model capable of predicting Runtime and I-V Performance" IEEE transactions on Energy conversions[9].A simplified model neglecting the effects of self discharge, cycle number, temperature in low power LI-ion batteries was presented. Liao Chenglin, Li Huiju,
Wang Lifang Institute of Electrical Engineering Chinese Academy of Sciences Beijing, China presented a paper in 2009 "A Dynamic Equivalent Circuit Model of LiFePO4 Cathode Material for Lithium Ion Batteries on Hybrid Electric Vehicles "-Journal of Power Sources, Elsevier[10].In this paper, the dynamic characteristics of the batteries are investigated over different current profiles. A dynamic equivalent circuit model was also proposed to describe the dynamic characteristics of the batteries. In the year 2004 the "Modelling of lithium ion cells - A simple equivalent-circuit model approach" Bor Yann Liaw, Ganesan Nagasubramanian, Rudolph G. Jungst, Daniel H. Doughty [11] presented an equivalentcircuit-based battery model, capable of simulating charge and discharge behaviour of lithium-ion batteries ( $\mathrm{LiB})$. The model, although simple in concept, can simulate complex discharge behaviour with high fidelity. "A dynamic lithium-ion battery model considering the effects of temperature and capacity fading" O. Erdinc, B. Vural and M. Uzunoglu, Member, IEEE (2009) [12]. In this paper, a dynamic model of lithium-ion battery has been developed with MATLAB/Simulink ${ }^{\circledR}$ in order to investigate the output characteristics of lithium-ion batteries. Dynamic simulations are carried out, including the observation of the changes in battery terminal output voltage under different charging/discharging, temperature and cycling conditions, and the simulation results are compared with the results obtained from several recent studies. In 2006 J.J. Esperilla, J. Fe'lez, G. Romero, A. Carretero ETS Ingenieros Industries, Universidad Polite' cnica de Madrid (UPM), Jose Gutierrez Abascal, 2, 28006 Madrid, Spain in their paper [13]."A model for simulating a lead-acid battery using bond graphs" presented a model of a lead-acid battery developed with bond graphs. The bond graph structure is used to reproduce the behaviour of reversible electrochemical cells in charging conditions or in discharging conditions. The work presented here has been applied to the particular case of standard $12 \mathrm{~V}$ lead-acid battery, which is widely used in automotive industries, traction, and electrical or hybrid vehicles.

In this paper the internal parameters are extracted for Sony make, $5 \mathrm{~V}, 2 \mathrm{Ahr}$ Lithium ion battery and a modelling has been done for its capacity fading after 50 cycles of operation. Covariance Matrix Adaptation Evolutionary Strategy (CMA-ES) has been applied to optimize the internal circuit parameters of a battery, under different charging and discharging rates. The algorithm results are compared with the catalogue values given by the manufacturer for validation. The obtained battery characteristics and capacity fading results coincide with the measured (manufacturer catalogue) characteristics in this paper.

The paper is organized as follows. Section 2 describes the proposed battery model. Evolutionary algorithm is explained in Section 3. The details of battery parameter extraction and capacity fading are discussed in results and discussion of Section 4. The model validation has been done in the Section 4 by comparing the proposed model result with manufactures' data. Section 5 concludes the research work. 


\section{Battery model}

An Electric Equivalent Circuit for Li-ion battery [6, 7] is shown in Fig. 1. The charging and discharging characteristics are exponentially increasing and decreasing with respect to rate of charging or discharging. Hence a polynomial equation with exponential function has been used to represent the characteristics of battery.

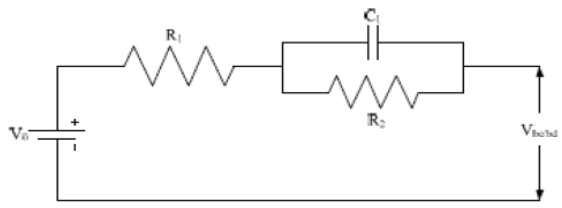

Fig. 1 Electric Equivalent Circuit for Li ion battery

\subsection{Internal battery parameters}

The general polynomial equations for the calculation of internal parameters of the battery are given below [3]. A set of 31 coefficients are used in the above set of equations to represent the parameters in terms of polynomial equations. The battery parameters can be derived by replacing $\mathrm{x}$ and $\mathrm{y}$ with $\mathrm{Cr}$ and $\mathrm{SOC}_{\mathrm{cr}}$, for charging process, whereas for discharging process with $\mathrm{Dr}$ and $\left(1-\mathrm{DOD}_{\mathrm{cr}}\right)$.

$$
\begin{aligned}
& R_{1}=\left(a_{1}+a_{2} x+a_{3} x^{2}\right) e^{-a_{4} y}+ \\
& \left(a_{5}+a_{6} x+a_{7} x^{2}\right) \\
& R_{2}=\left(a_{8}+a_{9} x+a_{10} x^{2}\right) e^{-a_{11} y}+ \\
& \left(a_{12}+a_{13} x+a_{14} x^{2}\right) \\
& C=-\left(a_{15}+a_{16} x+a_{17} x^{2}\right) e^{-a_{18} y}+ \\
& \left(a_{19}+a_{20} x+a_{21} x^{2}\right) \\
& V_{0}=\left(a_{22}+a_{23} x+a_{24} x^{2}\right) e^{-a_{25} y}+ \\
& \left(a_{26}+a_{27} y+a_{28} y^{2}+\right. \\
& \left.\left.a_{29} y^{3}\right)-a_{30} x+a_{31} x^{2}\right)
\end{aligned}
$$

where $\mathrm{R}_{1}, \mathrm{R}_{2}, \mathrm{C}$ are internal parameters and are represented in terms of polynomial equations; $V_{0}$ is the voltage between the terminals of battery in open circuit condition.

\subsection{Battery charging/discharging voltage}

The battery terminal voltage for charging/discharging scenario with respect to time under constant current is given in Eq. 5 and Eq. 6 [3]. The accurate behaviour of any type of battery can be represented by Eq. 5 and Eq. 6 , if the parameters are accurately extracted. The nonlinear behaviour can be captured by these equations

$$
\begin{aligned}
& V_{C_{i}}^{C}=\left(\left(\frac{Q_{r}}{C}+I_{c} R_{2}\right) \exp \left(-\frac{t_{c}}{R_{2} C}\right)\right)+ \\
& V_{0}-\left(I_{c}\left(R_{1}+R_{2}\right)\right)
\end{aligned}
$$

$$
\begin{aligned}
& V_{d_{j}}^{C}=\left(\left(\frac{Q_{r}}{C}+I_{d} R_{2}\right) \exp \left(-\frac{t_{d}}{R_{2} C}\right)\right)+ \\
& V_{0}-\left(I_{d}\left(R_{1}+R_{2}\right)\right)
\end{aligned}
$$

where $Q_{r}$ is the remaining capacity of the battery; $t_{c}, I_{c}, t_{d}$ and $I_{d}$ are charge time, charge current, discharge time and discharge current, respectively.

\subsection{Charge/Discharge rate and SOC calculations}

The charge or discharge rate algorithm is used to determine the amount of energy stored or extracted from the battery. The $\mathrm{C}_{\mathrm{r}}$ and $\mathrm{SOC}_{\mathrm{cr}}$ of the battery vary depending on the present condition of the battery. The battery status is checked and the current charge rate $\left(\mathrm{C}^{\mathrm{crt}}\right)$ of the battery is calculated by the control algorithm developed inside the battery. User defined $\mathrm{C}_{\mathrm{r}}$ limit $\left(\mathrm{C}^{\mathrm{lmt}}\right)$ and initial battery $\mathrm{SOC}\left(\mathrm{SOC}_{\mathrm{ini}}\right)$ are also taken into account .The $C_{r}{ }^{\text {crt }}$ and $D r^{c r t}$ of the battery can be calculated from Eq. (7) \& (8).

$$
\begin{gathered}
C_{r}=C_{r}^{c r t}=\frac{I_{c}}{Q_{r}} \\
D_{r}=D_{r}^{c r t}=\frac{I_{d}}{Q_{r}}
\end{gathered}
$$

The above are calculated based on present status of the battery, which is the ratio of current and remaining capacity of the battery. The minimum of charge rate based on the $\mathrm{C}^{\mathrm{lmt}}$ and $\mathrm{C}^{\mathrm{crt}}$ charge are found by the algorithm to regulate the charge current of the battery. This type of control algorithm is used for discharging scenario. The $\mathrm{SOC}_{\mathrm{cr}}$ and $\mathrm{DOD}_{\mathrm{cr}}$ can be calculated from Eq. 9 to Eq. 10.

$$
\begin{gathered}
S O C_{c r}=S O C_{i n i}+\left(\frac{I_{c} \Delta t_{c}}{Q_{r} 3600}\right) \\
D O D_{c r}=D O D_{i n i}+\left(\frac{I_{d} \Delta t_{c}}{Q_{r} 3600}\right)
\end{gathered}
$$

Where, $\mathrm{SOC}_{\mathrm{ini}}$ is the initial SOC of the battery; $\mathrm{SOC}_{\max }$ and $\mathrm{DOD}_{\max }$ are the maximum user defined SOC and DOD limits respectively.

\section{Capacity Fade Model}

The energy storage capacity of battery decreases due to frequent charging or discharging. In this paper, the capacity loss is calculated at an interval of 10 cycles from $50^{\text {th }}$ cycle based on variable charge or discharge rate. A cycle is defined as an interval during which the battery charges till $S O C_{\max }$ and discharges up to $D O D_{\max }$. When the battery experiences different charge and discharge cycles, it fails to meet its performance thus reducing its life expectancy. Therefore, it is required to study the capacity fade to quantify the capacity loss with respect to number of cycles. 


\subsection{Battery power and processed energy}

The battery power for charging $\left(P_{c}\right)$ and discharging $\left(P_{d}\right)$ scenario are given in Eq. (11) and Eq. (12). The amount of stored energy $\left(E_{\text {stor }}\right)$ during charging process depends on increase in $V_{c i}^{C}$ and $S O C_{c r}$, which is given in Eq. (13).

$$
\begin{aligned}
& P_{c}=V_{c_{i}}^{c} I_{c} \\
& P_{d}=V_{d_{j}}^{C} I_{d} \\
& E_{\text {stor }}=V_{c_{i}}^{C} Q_{r} \Delta S O C_{c r}
\end{aligned}
$$

where $\triangle S O C_{c r}$ is the change in current $S O C_{c r}$. The processed energy $\left(P E_{c}\right)$ for charging scenario is given in Eq. (14).

$$
P E_{c}=\sum E_{\text {stor }}
$$

The available energy $\left(E_{\text {avail }}\right)$ in the battery during discharging process decreases $V_{d i}^{c}$ with increase in $D O D_{c r}$, which can be calculated using Eq. (15).

$$
E_{\text {avail }}=V_{d_{j}}^{C} Q_{r} \Delta D O D_{c r}
$$

where, $\triangle D O D_{c r}$ is the change in current $D O D_{c r}$. The processed energy $\left(P E_{d}\right)$ for discharging scenario is given in Eq. (16).

$$
P E_{d}=\sum E_{\text {avail }}
$$

The total processed energy $\left(E_{\text {total }}\right)$ of the battery in a cycle is calculated using Eq. (17).

$$
E_{\text {total }}=\sum P E_{c}+P E_{d}
$$

Eq. (11) to Eq. (17) represents the real-time performance of the battery during charging and discharging process. Simulations are done based on these equations for the developed battery model and have been validated with the manufacturers' catalogue.

\subsection{ABCD Parameters of capacity fade model}

Capacity fade of the battery can be quantified using Arrhenius equation for Constant charge or discharge rate [16]. The mathematical model used to quantify the capacity fade is based on Arrhenius equation and is given in Eq. $(18)[14,15]$. Here, $A$ is pre-exponential factor, $Q_{l}$ represents the capacity loss (Ah), $E_{a}$ denotes the activation energy $(\mathrm{J}), R$ is the gas constant $T$ is temperature $(\mathrm{K}), z$ is the adjustable factor.

$$
Q_{l}=A \exp \left(-\frac{E_{a}}{R T}\right) t^{2}
$$

Eq (18) is used for calculating capacity fade under constant $C_{r}$. However, in real-time grid support, the charge or discharge rate of the battery would vary with respect to peak power demand and node voltage variations. This causes capacity loss of the battery. In realtime, the batteries charge or discharge rate varies with respect to variable node voltage conditions, $S O C_{c r}$ or $D O D_{c r}$ limits, and temperature and also on the system constraints and user preferences. Due to this, the capacity fades changes with respect to the varying $C_{r}, D_{r} \mathrm{SOC}$,

DOD limits and temperature. To account this loss, a mathematical model is developed in this work to predict the capacity fade at different charge and discharge rate. The total capacity fade can be quantified and written in terms of nominal capacity $(\mathrm{Q}), C_{r}, D_{r}, P E_{c}, P E_{d}$, preexponential $(A, B)$ and adjustable $(C, D)$ factors, gas constant $(R)$ and temperature $(T)$ which is given in Eq. (19). In Eq. (19), $Q_{l k}^{C}$ denotes calculated capacity loss and $Q_{r}$ the remaining capacity of the battery can be calculated from Eq. (20).

$$
\begin{aligned}
& Q_{l_{k}}^{c}=A \exp \left(-\frac{C P E_{c} Q_{r} C_{r} S O C_{c r}}{R T}\right)+ \\
& B \exp \left(-\frac{D P E_{d} Q_{r} D_{r} D O D_{c r}}{R T}\right)
\end{aligned}
$$

$$
Q_{r}=Q-Q_{l_{k}}^{c}
$$

where, $Q_{r}$ is the remaining capacity of the battery which is u'pdated value of the capacity loss at every cycle. The pre-exponential and adjustable factors are calculated by CMAES. The capacity loss characteristics have been optimized by the fitness function $\left(F^{\prime}(x)\right) . \quad F^{\prime}(x)$ is maximized, when the difference between the measured (catalogue value) $(Q l k M)$ and calculated $(Q l k C)$ capacity loss is minimized. The mathematical representation of $F^{\prime}(x)$ is given in Eq. (21).Simulations are done to verify the developed capacity fade model and have been validated with manufacturers' catalogue.

$$
F^{\prime}(x)=\frac{1}{1+\sum_{k=1}^{n}\left(Q_{l_{k}}^{M}-Q_{l_{k}}^{C}\right)}
$$

The battery manufacturers' charge/discharge rate characteristics have been extracted using the mathematical equations described in this Section. The control algorithm decides the amount of energy stored into the battery or extracted from the battery based on the cur- rent energy status and user defined $C_{r} / D_{r}$ limit of the battery. The input of the control algorithm are charge/discharge current, SOC/DOD limits and polynomial coefficients for charging/discharging scenario $\left(a_{1}-a_{31}\right)$. Based on these inputs, the BM calculates $S O C_{c r} / D O D_{c r}$, charge/discharge voltage $(V C / V C)$, current $\left(I_{c} / I_{d}\right)$, power $\left(P_{c} / P_{d}\right)$ and energy $\left(P E_{c} / P E_{d}\right)$. Capacity loss is calculated using energy of the battery, pre-exponential factor, gas constant, temperature and adjustable factor.

\subsection{Fitness function}

Battery parameters are extracted with population based search using bio inspired evolutionary algorithms based optimization technique. The aim of using evolutionary algorithms is due to the fact that it needs only manufacturers $C_{r}$ and $D_{r}$ Characteristics for giving polynomial coefficient in relatively less iteration. Also, evolutionary algorithm is more flexible in extracting the battery parameters with any initial values, while other analytical techniques are not capable of obtaining feasible 
solutions. It is easy to understand and can be optimized using fitness function. Different types of manufacturers' data of Li-ion batteries are considered for extraction purpose. The main objective of the algorithm is to optimize the battery parameters polynomial coefficients $\left(a_{1}-a_{31}\right)$ to evaluate the equations given in Eq. (1) to Eq. (6) and optimize the adjustable factors and exponential factors $(A, B, C, D)$ to evaluate the equations given in Eq (19). The requirement after generation of random solution set is the measure of quality of solution set. This can be achieved by establishing a fitness function $\mathrm{F}(\mathrm{x})$, which is rated by each solution according to its fitness. The difference between the measured $\left(\boldsymbol{V}_{\boldsymbol{c}_{\boldsymbol{i}}}^{\boldsymbol{M}}\right.$ or $\left.\boldsymbol{V}_{\boldsymbol{d}_{\boldsymbol{j}}}^{\boldsymbol{M}}\right)$ and calculated voltage for charging and discharging cases $\left(\boldsymbol{V}_{\boldsymbol{c}_{\boldsymbol{i}} \text { or }}^{\boldsymbol{c}} \boldsymbol{V}_{\boldsymbol{d}_{\boldsymbol{j}}}^{\boldsymbol{c}}\right)$ are determined with Eq.(5) and Eq.(6) respectively. Based on the calculated fitness value, the best list gets updated and the lowest fitness is discarded. The mathematical formulation of fitness function $f(x)$ for charging and discharging scenario is in Eq. (11) - (12).

$$
\begin{array}{r}
\text { Minimize } \mathrm{f}(\mathrm{x}) \\
f(x)=\left\{\begin{array}{c}
\sum_{i=0}^{n}\left|\left(V_{c_{i}}^{M}-V_{c_{i}}^{C}\right)\right| \\
\sum_{j=0}^{m}\left|\left(V_{d_{j}}^{M}-V_{d_{j}}^{C}\right)\right|
\end{array}\right.
\end{array}
$$

\section{Covariance Matrix Adaptation Evolution Strategy}

A recent approach to adapting the search direction is Covariance Matrix Adaptation Evolution Strategy (CMAES). It is important property is invariance against the linear transformations in the continuous search space. CMA-ES outperformed its other similar learning algorithms in CEC2005 benchmark functions [16, 17] and BBOB-2009 benchmark functions [18]. Hence, an attempt is made to optimize the Battery Modelling design using CMA-ES in this research work. CMA learns all pair wise dependencies between the variables and increases the probability to repeat the successful steps. One evolution path, enhances CMA procedure in place of single successful search steps and facilitates possibly much faster increase of favourable directions. CMA identifies the function landscape which is convexquadratic one with the concept of $\operatorname{Hessian}$ matrix $(H)$. The CMA-ES estimates the inverse Hessian matrix $\left(H^{-1}\right)$ in the form of a covariance matrix of the search distribution within an iterative procedure. The aim of CMA is to approximate the matrix, ' $H$ ' and to closely suit the search direction to the contour lines of the objective function to be optimized [19]. The CMA influences the scale of the distribution. Nevertheless, additional step-size, control is necessary [20]. So, the step size update is also introduced to enhance the scaling adaptation and in particular to facilitate the increase of distribution spread, which is very difficult with CMA only. Step size adaptation aims to make consecutive movements of the distribution mean orthogonal in expectation and prevents the premature convergence.

\section{Results and Discussion}

The Li-ion batteries manufactured by SONY US18650, $5 \mathrm{~V}, 2 \mathrm{Ahr}$ considered for parameter extraction problem. For Li-ion battery, SOC verses battery terminal voltage for charging, and SOC verses battery terminal voltage for discharging at different charge/discharge rates for various cycles are taken from manufacturer catalogue. To obtain the parameters $\left(\mathrm{a}_{1}-\mathrm{a}_{31}\right)$, measure the charging voltage, and discharging voltage at different charge/discharge rate. The parameters for charging and discharging characteristics are calculated from GA and CMA-ES. The mathematical formulation of fitness function for obtaining one set of the parameters for charging and discharging scenario are given in Equations $(11,12)$. The results of established mathematical model for battery at different charge/discharge rate are discussed in this section. Battery charge/discharge rate characteristics were obtained for the cycles 50 to 100 in the intervals of 10 cycles and compared with the measured (manufacturer catalogue) data.

The plots of charge curves obtained for charge/discharge for cycle No 50 are calculated using Equation $(5,6)$. The experimental data's are measured from the battery manufacturer's catalogue. It is evident from the plots that the simulated curves are in close agreement with the measured data for CMA-ES. The polynomial coefficients obtained by conducting simulations for charging and discharging characteristics after 50,60,70,80 and 100 are listed in the Table 1 . The results for the capacity fading i.e pre adjustable factors(A,B) ,Adjustable Factors(C,D) and the Capacity of the battery fading have been listed in Table 2. The plot for the cycle Vs remaining capacity of the battery is drawn in Fig 5. From the figure it can be seen that the capacity of the battery reduces as the number of cycle increases. The remaining capacity of the battery after each cycle can be found from this graph.

\subsection{Sony US18650 battery charging characteristics after 50 cycles}

The variation of charging/discharging voltage with respect to increasing $\mathrm{SOC}_{\mathrm{cr}}$ and $\mathrm{DOD}_{\mathrm{cr}}$ are shown in Figures 1 and 2 respectively by CMA-ES method. The simulations are observed for $\mathrm{SOC}_{\mathrm{cr}} / \mathrm{DOD}$ cr from 0$100 \%$. The difference between the calculated and catalogue values varies from 0 to $22.5 \mathrm{mV}$. It is clear from the plot that the battery can be discharged only upto $90 \%$ of total capacity. Beyond this the voltage of the battery rapidly decreases. So the maximum DOD for the battery at $0.5 \mathrm{Dr}$ is $90 \%$. The fitness Value for charge and discharge rate is calculated using CMA-ES as $8.6482 \mathrm{~V}$ and $8.1738 \mathrm{~V}$ respectively.

\subsection{Sony US18650 battery charging characteristics after 60 cycles}

The variation of charging/discharging voltage with respect to increasing $\mathrm{SOC}_{\mathrm{cr}}$ and $\mathrm{DOD}_{\mathrm{cr}}$ are in Figures 3 and 4 respectively by CMA-ES method. The simulations are observed for $\mathrm{SOC}_{\mathrm{cr}} / \mathrm{DOD}$ cr from $0-100 \%$. The 
différence between the calculated and catalogue values varies from 0 to $22.5 \mathrm{mV}$. It is clear from the plot that the battery can be discharged only upto $90 \%$ of total capacity. Beyond this, the voltage of the battery rapidly decreases. So the maximum DOD for the battery is $90 \%$. The fitness Value for charge and discharge rate is calculated using CMA-ES as $393.2175 \mathrm{~V}$ and $8.1738 \mathrm{~V}$ respectively. The measured energy that can be discharged from the battery are $5.770 \mathrm{Wh}$ and $5.7881 \mathrm{Wh}$

\section{CONCLUSION}

In this work an accurate circuit based battery modeling have been done for a real type of battery available in market. The novelty of the model is that the optimization procedure is done by Real GA while binary GA has been used earlier. The battery polynomial coefficients are extracted using CMAES based optimization methods for Sony Us 18650 battery after 50 cycles in an interval of 10 cycles upto 100 cycles. The results has been compared with the measured values given by the manufacturer and the results calculated by the equations are accurate. Five cycles charge rate and discharge rate characteristics of Sony US 18650 battery have been obtained and compared with manufacturer's data for validation. The capacity fading of the battery after 50 cycles has been modeled at an interval of 10 cycles up to 100 cycles and graph has been drawn which accurately represents the catalog values. The proposed battery models are simple and it accurately represents the measured (catalogue value) charge and discharge curves of the manufacturers data sheets. The simulation and measured (catalogue value) results are in good agreement. It has been seen that the methodology presented in this work extracts accurate results and this can be extended to obtain the Capacity fading of different batteries after every cycle of charging and discharging. Future works can be carried for modeling of other types of batteries with the same procedures.

\section{References}

1. https://factly.in/indias-per-capita-electricity-consumptionlowest-among-brics-nations/“Energy Calculation per Capita," $\mathrm{http}: / / \mathrm{www}$.indexmundi.com/g/r.aspx/ $\mathrm{v}=81000$

2. "Electricity Generation in India and Power Sector at a Glance ALL INDIA" http://en.wikipedia.org/wiki/Electricity sector in Indiaandhttp:// www. powermin.nic.in/ Indian electricity scenario/introduction.htm.

3. C. M. Shepherd, "Design of primary and secondary cells - part 2. An equation describing battery discharge," J Electroche. Soc., vol. 112, pp. 657-664, Jul. 1965.

4. O. Tremblay, L.-A. Dessaint, and A.-I. Dekkiche, "A generic battery model for the dynamic simulation of hybrid electric vehicles," IEEE Int. Vehicle Power and Propulsion Conf. (VPPC'07), pp. 284-289, Sept. 2007.

5. E. Sortomme and M. El-Sharkawi, "Optimal combined bidding of Vehicle-to-Grid ancillary services," IEEE Trans. on Smart Grid, vol. 3, no. 1, pp. 70-79, Mar. 2012.

6. "Parameter extraction of Battery Models Using Multi objective Optimization Genetic Algorithms"- Praveen kumar, Pavol Bauser -14 th International Power Electronics and motion Control Conference -2010.
7. "Mathematical Modeling of Li-Ion Battery for Charge/Discharge Rate and Capacity Fading Characteristics using Genetic Algorithm Approach." And published in IEEE journal 2012

8. Mathematical Modeling of Li-Ion Battery Using Genetic Algorithm Approach for V2G Applications" , Kannan Thirugnanam, Student Member, IEEE, Ezhil Reena Joy T. P., Student Member, IEEE, Mukesh Singh, Student Member, $I E E E$, and Praveen Kumar, Member, IEEE- IEEE transactions on Energy conversions-2014 K. Deb, "Optimization for engineering design: Algorithms and examples," Prentice Hall, India, 1998

9. Min Chen,Student Member IEEE and Gabriel A.RinconMora,Senior Member ,IEEE published a paper "Accurate Electrical Battery Model capable of predicting Runtime and IV Performance"IEEE trans on Energy conversions

10. Liao Chenglin, Li Huiju, Wang Lifang Institute of Electrical Engineering Chinese Academy of Sciences Beijing, China presented a paper in 2009 "A Dynamic Equivalent Circuit Model of LiFePO4 Cathode Material for Lithium Ion Batteries on Hybrid Electric Vehicles "-Journal of Power Sources, Elsveir

11. Bor Yann Liaw, Ganesan Nagasubramanian, Rudolph G. Jungst, Daniel H. Doughty, "Modeling of lithium ion cellsA simple equivalent-circuit model approach

12. "A dynamic lithium-ion battery model considering the effects of temperature and capacity fading "O. Erdinc, B. Vural and M. Uzunoglu, Member, IEEE (2009)

13. J.J. Esperilla, J. Fe'lez *, G. Romero, A. Carretero ETS Ingenieros Industries, Universidad Polite'cnica de Madrid (UPM), Jose Gutierrez Abascal, 2, 28006 Madrid, Spain

14. P. Ramadass, B. Haran, R. White, and B. N. Popov, "Mathematical modeling of the capacity fade ofli-ion cells," $J$ Power Sources, vol. 123, pp. 230-240, 2003.

15. C. M. Shepherd, "Design of primary and secondary cells - part 2. an equation describing battery discharge,"J Electroche. Soc., vol. 112, pp. 657-664, Jul. 1965.

16. Suganthan, P, Hansen, N, Liang, JJ, Deb, K, Chen, YP, Auger, A, \& Tiwari, S 2005, 'Problem, definitions and evaluation Criteria for the CEC 2005', in Special Session on RealParameter Optimization, Technical report, Nanyang Technological University, Singapore.

17. Hansen, N, Auger, A, Ros, R, Finck, S, \& Pošík, P 2010, 'Comparing results of 31 algorithms from the black-box optimization benchmarking BBOB-2009', Proceedings of the twelth annual conference companion on Genetic and evolutionary computation, GECCO, pp. 1689-1696.

18. Hansen, N, \& Ostermeier, A 2001, 'Completely de randomized self-adaptation in evolution strategies', Evolutionary Computation, 9(2) 159-195.

19. Hansen, N, Auger, A, Ros, R, Finck, S, \& Pošík, P 2010, 'Comparing results of 31 algorithms from the black-box loptimization benchmarking BBOB-2009', Proceedings of the twelth annual conference companion on Genetic and evolutionary computation, GECCO, pp. 1689-1696

20. Hansen, N 2006, 'The CMA evolution strategy: a comparing review', in towards a new evolutionary computation, Springer Berlin, pp. 75-102.

21. Liao Chenglin, Li Huiju, Wang Lifang Institute of Electrical Engineering Chinese Academy of Sciences Beijing, China presented a paper in 2009 "A Dynamic Equivalent Circuit Model of LiFePO4 Cathode Material for Lithium Ion Batteries on Hybrid Electric Vehicles "-Journal of Power Sources, Elsevier.

22. "Modeling of lithium ion cells-A simple equivalent-circuit model approach Bor Yann Liaw, Ganesan Nagasubramanian, Rudolph G. Jungst, Daniel H. Doughty. 


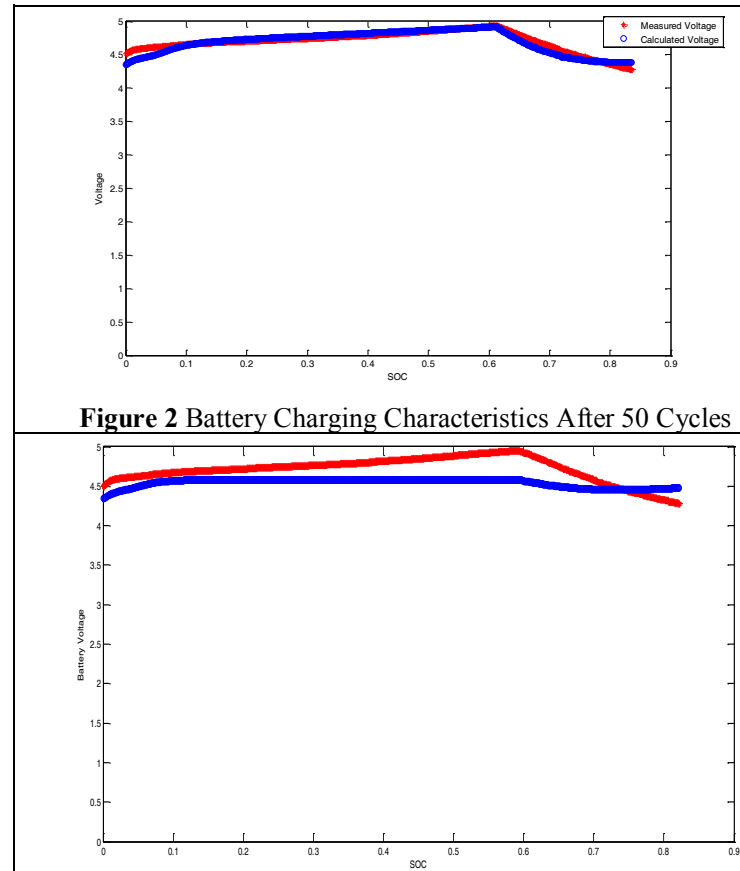

Figure 4 Battery Charging Characteristics After 60 Cycles

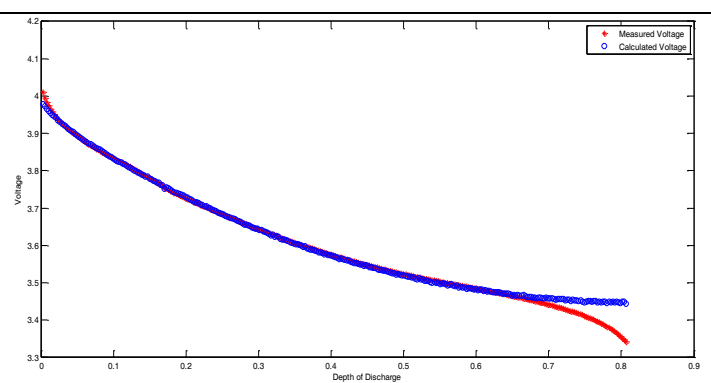

Figure 3 Battery Discharging Characteristics After 50 Cycles.

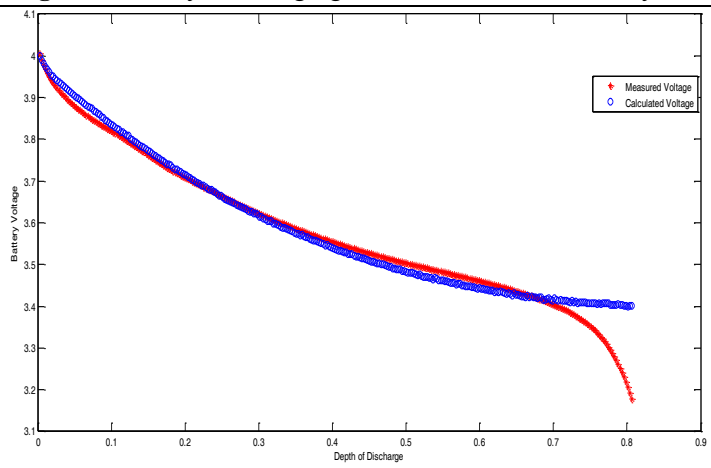

Figure 5 Battery Discharging Characteristics After 60 Cycles.

Table 1. Capacity Polynomial Coefficients for charging/discharging scenario

\begin{tabular}{|c|c|c|c|c|c|c|c|c|c|c|c|c|}
\hline \multirow[b]{2}{*}{ Coeff } & \multicolumn{5}{|c|}{ Charging Scenario Cycle No } & \multicolumn{7}{|c|}{ Discharging Scenario Cycle No } \\
\hline & 50 & 60 & 70 & 80 & 90 & 100 & $\mathbf{5 0}$ & 60 & 70 & 80 & 90 & 100 \\
\hline$a_{1}$ & 0.000 & 0.133 & 0.000 & 0.000 & 0.000 & 0.000 & 0.000 & 0.000 & 0.000 & 0.000 & 0.000 & 0.021 \\
\hline$\overline{a_{2}}$ & 0.000 & 0.000 & 0.370 & 0.050 & 0.000 & 0.000 & 0.000 & 0.000 & 0.000 & 0.000 & 0.000 & 0.000 \\
\hline$\overline{a_{3}}$ & 0.339 & 0.000 & 0.000 & 0.000 & 0.000 & 0.534 & 0.000 & 0.000 & 0.027 & 0.000 & 0.000 & 0.000 \\
\hline$a_{4}$ & 44.305 & 50.000 & 46.010 & 3.165 & 50.000 & 48.448 & 26.573 & 42.816 & 26.731 & 50.000 & 27.818 & 50.000 \\
\hline$\overline{a_{5}}$ & 0.000 & 0.000 & 0.000 & 0.038 & 0.000 & 0.000 & 0.000 & 0.000 & 0.000 & 0.000 & 0.000 & 0.000 \\
\hline$\overline{a_{6}}$ & 0.000 & 0.000 & 0.000 & 0.000 & 0.000 & 0.000 & 0.000 & 0.000 & 0.000 & 0.000 & 0.000 & 0.000 \\
\hline$\overline{a_{7}}$ & 0.000 & 0.000 & 0.000 & 0.000 & 0.000 & 0.000 & 0.000 & 0.000 & 0.000 & 0.000 & 0.000 & 0.000 \\
\hline$\overline{a_{8}}$ & 0.000 & 0.226 & 0.008 & 0.307 & 0.163 & 0.067 & 0.000 & 0.000 & 0.000 & 0.000 & 0.000 & 0.173 \\
\hline$a_{9}$ & 0.303 & 0.207 & 0.007 & 0.164 & 0.000 & 0.000 & 0.000 & 0.000 & 0.000 & 0.000 & 0.000 & 0.149 \\
\hline$a_{10}$ & 0.517 & 0.000 & 0.000 & 0.418 & 0.000 & 0.201 & 0.000 & 0.000 & 0.000 & 0.000 & 0.000 & 0.000 \\
\hline$a_{11}$ & 29.006 & 38.224 & 26.828 & 46.608 & 50.000 & 16.703 & 43.329 & 44.710 & 50.000 & 0.000 & 49.640 & 49.061 \\
\hline$a_{12}$ & 0.000 & 0.004 & 0.000 & 0.000 & 0.000 & 0.042 & 0.000 & 0.000 & 0.059 & 0.799 & 0.000 & 0.000 \\
\hline$a_{13}$ & 0.000 & 0.000 & 0.035 & 0.000 & 0.000 & 0.000 & 0.000 & 0.859 & 0.000 & 0.000 & 0.000 & 0.000 \\
\hline$a_{14}$ & 0.000 & 0.000 & 0.000 & 0.137 & 0.000 & 0.286 & 0.969 & 0.000 & 0.733 & 0.000 & 0.858 & 0.000 \\
\hline$a_{15}$ & 520.021 & 1000 & 0.000 & 95.470 & 394.12 & 332.68 & 354.80 & 1000 & 812.11 & 350.27 & 449.01 & 0.000 \\
\hline$a_{16}$ & 163.403 & 453.90 & 948.98 & 329.11 & 0.000 & 0.000 & 604.828 & 1000.000 & 246.990 & 42.707 & 381.947 & 340.079 \\
\hline$a_{17}$ & 281.682 & 1000.000 & 183.508 & 863.322 & 0.000 & 256.458 & 0.000 & 152.764 & 0.000 & 538.446 & 173.985 & 358.668 \\
\hline $\mathrm{a}_{18}$ & 11.338 & 50.000 & 50.000 & 12.776 & 41.635 & 46.509 & 15.217 & 50.000 & 14.753 & 24.148 & 16.643 & 50.000 \\
\hline$a_{19}$ & 1000.000 & 853.942 & 1000.000 & 757.967 & 353.886 & 613.630 & 1000.000 & 1000.000 & 993.682 & 1000.000 & 1000.000 & 776.075 \\
\hline$a_{20}$ & 897.165 & 1000.000 & 0.000 & 873.389 & 651.093 & 0.000 & 1000.000 & 1000.000 & 995.364 & 1000.000 & 1000.000 & 766.410 \\
\hline$a_{21}$ & 630.715 & 945.054 & 1000.000 & 1000.000 & 1000.000 & 547.346 & 1000.000 & 1000.000 & 1000.000 & 1000.000 & 1000.000 & 771.229 \\
\hline$a_{22}$ & 0.168 & 0.076 & 0.000 & 1.000 & 0.081 & 0.182 & 0.000 & 0.042 & 0.041 & 0.000 & 0.000 & 0.540 \\
\hline$a_{23}$ & 0.000 & 0.725 & 0.000 & 0.947 & 0.776 & 0.631 & 0.000 & 0.000 & 0.000 & 0.000 & 0.000 & 0.000 \\
\hline $\mathrm{a}_{24}$ & 0.138 & 0.000 & 0.000 & 0.443 & 0.000 & 0.000 & 0.000 & 0.000 & 0.000 & 0.000 & 0.000 & 0.679 \\
\hline$a_{25}$ & 0.000 & 0.000 & 50.000 & 0.000 & 0.000 & 0.000 & 50.000 & 50.000 & 39.645 & 35.153 & 29.595 & 1.436 \\
\hline$a_{26}$ & 3.834 & 4.417 & 4.443 & 2.217 & 3.802 & 3.616 & 5.000 & 5.000 & 5.000 & 5.000 & 5.000 & 2.822 \\
\hline$a_{27}$ & 0.451 & 0.000 & 0.000 & 0.561 & 0.420 & 0.000 & 0.848 & 0.711 & 0.623 & 0.590 & 0.660 & 0.055 \\
\hline $\mathrm{a}_{28}$ & 0.000 & 0.000 & 0.000 & 0.751 & 0.014 & 0.710 & 0.000 & 0.000 & 0.000 & 0.000 & 0.000 & 0.114 \\
\hline $\mathrm{a}_{29}$ & 0.000 & 0.000 & 0.000 & 0.208 & 0.014 & 0.000 & 0.000 & 0.000 & 0.000 & 0.000 & 0.000 & 0.256 \\
\hline$a_{30}$ & 0.000 & 1.000 & 0.371 & 0.321 & 0.943 & 0.000 & 1.000 & 1.000 & 1.000 & 1.000 & 1.000 & 0.688 \\
\hline $\mathrm{a}_{31}$ & 1.000 & 0.536 & 1.000 & 0.349 & 0.363 & 0.000 & 0.000 & 0.000 & 0.000 & 0.000 & 0.000 & 0.594 \\
\hline
\end{tabular}

Table 2. Capacity Fading

\begin{tabular}{|c|l|r|l|l|l|l|}
\hline Cycle & \multicolumn{1}{|c|}{$\mathbf{5 0}$} & $\mathbf{6 0}$ & $\mathbf{7 0}$ & $\mathbf{8 0}$ & $\mathbf{9 0}$ & \multicolumn{1}{|c|}{$\mathbf{1 0 0}$} \\
\hline A & 0.17 & 0.114 & 0.114 & 0.174 & 0.264 & 0.186 \\
\hline B & 0.152 & 0.235 & 0.3 & 0.259 & 0.224 & 0.329 \\
\hline C & 5.994 & 5.089 & 3.277 & 5.28 & 7.637 & 4.454 \\
\hline D & 7.797 & 4.469 & 2.723 & 7.784 & 8.244 & 7.357 \\
\hline
\end{tabular}

\footnotetext{
* Corresponding author: tamilselvi.manjuraj@gmail.com
} 


\begin{tabular}{|c|c|c|c|c|c|c|}
\hline Q & \\
\hline Qmeas)Ah & 1.685 & 1.656 & 1.589 & 1.576 & 1.523 & 1.495 \\
\hline calc) Ah & 1.685 & 1.656 & 1.589 & 1.576 & 1.523 & 1.495 \\
\hline
\end{tabular}

